Historic, Archive Document

Do not assume content reflects current scientific knowledge, policies, or practices. 

FALL 1932

TRADE LIST

Terms of sale: All goods offered subject to prior sale F.O.P. Springtield, Ohio.

Five per cent discount when cash accompanies order. No packing charges.

\section{America's Finest Peonies}

3 to 5 -eye divisions with well balanced root systems.

New Introductions

DR. J, H. NEELEY, Pure white...... JUDGE SNOOK, Pure white. GOOD'S GEM (Jap.), Pure white. GOOD'S IDEAL, Creamy white...
$\$ 25.00$ each

$\$ 25.00$ each

$\$ 25.00$ each

$\$ 25.00$ each
DARLIYG OMIXE, Delicate pink, ...., \$25.00 pach ANNE NIELSEN, Delicate pink

MRS. J. II. PERKINS, Creamy white.

SWA. WIIITE (Single), Snow white.....
(825 00 each

$\$ 17.50$ cach

$\$ 5.00$ each

General List of Double Peonies

E-denotes Early, M-Midseason, L-Late

Rating

8.8 ADDIELAXCIIEA-Creamy white .........

7.7 ADELAIDE IIOLL1S Lilac white a.....

S.5 ADOLPHE ROISSEAU-1 M) Deep garnet

8.6 ALBERT CROYSSE-(L) Shell-pink

7.1 ALEXANDRE DIMAS - (E) Brilliant pink........

9.5 ALICE IIARDING - Pure white

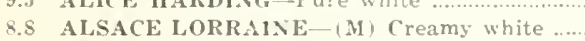

AMERICAN REA:TY M, Glowin. red

ANDRE LACRIES - (L) Solferino-re!

8.1 ARLEQiI Purplish pink....

8.1 ASA GIRAY-.. M) Lilac

7.3 ATROSANGLINEA - (M) Rosy-magenta

Q.7 ALGUSTE DESSERT Crimson-Carmine

7.8 ALGUSTIN d'HOLR - (MI) Brilliant solferino-red

8.0 AURORE-Lilac-white

8.7 AVALANCHE-1 M1 Pure snow-white

9.0 BARONESS SCHROEDER - (I, ) Pure white

8.5 BAYADERE - (M) Creamy white

8.1 BENJAMIN FRAN゙KLINー-(M) Brilliant$$
\text { crir... }
$$

7.0 BERLIOZ-(L) Bright currant-red ..........................

BLAXCHE KING-Deen Pink

8.0 BOLLE DE NEIGE-(E) Milk-white

8.2 BRAND'S MAGNIFICENT (MI) Dark rerl CANARI-(II) Pure white

8.4 CANDELK - Delicate pink

7.1 CANDIDISSIMA-(E) Creamy-white

8.4 CHARLOT - Silvery lilac

7.8 CIIARLES McKELLIP-(E) Rich crimson

8.6 CHERIY HILL- IN Deep garnet

8.7 CLAIRE DUBOIS- L L ) Satiny-pink

8.5 (LEMENCEAU-(L) Carmine

9.1 CORNELIA SHAYLOR-IL) Shell-pink.

8.1 COL'RONNE D'OR-MI Snow-white

7.2 DE CANDOLLE- M Currant-red

7.6 DOCTEIR BOISDLVAL-(M) Dark red

8.1 DOCTOR H. BARNSBY (L) Crimson

7.7 DORCHESTER (L) Salmon pink.

7.8 DIC DE WELLINGTOX (M) Creamy white

\$.1 DLCHESS DE NFMOLRS - (E) Sulphur-white.

8.6 ED.MOND ABOtT-(M) Hydrangea pink

7.1 EDOLARD ANDRE-1 M, Velvety crimson.

EDI LIS SLPERBA-E, Bright clear-pink....

.5 EDWIN FORREST- $M$, Light red

9.1 EDWIN C. SIIAW - Shell-pink

8.7 E, J. SIIAYLOR Silvery-pink

7.8 E. G. IIILL-1M) Rich rerl

7.3 ELIE ('HEVALIEIR ( MI) Tyrian-rose

8.5 ELISA Hyitrangea nink 9.2 ELIZABETII BARRETT BROWNING (L)

ELLA CHRISTIANSEN-(MI) Deep pink

7.6 ELLA WIEFLER WILCOX - L Shell pink

$\checkmark .7$ ELWOOD PLEAS - M, Shell pink

\&.9 ENCHANTRESSE - (L) Creany-whit

२.0 ETTA - (L) Hydrangea-pink

\$.6 ELGENIE VEIRIEI - M, Delicat pink

8.9 EUNICE SIIAILOR-(M) Ivory white

9.3 FESTIVA MAXIMA-(E) Most popular white.

\begin{tabular}{|c|c|c|c|}
\hline $\begin{array}{l}\text { Per } \\
\text { Each }\end{array}$ & $\begin{array}{c}\text { Per } \\
12\end{array}$ & $\begin{array}{r}\text { Per } \\
100\end{array}$ & Rating \\
\hline \multirow[t]{2}{*}{$\$ 1.75$} & $\$ 17.50$ & & 8.4 FELIX CROUSSE-( 21$)$ Ruby-red . ... \\
\hline & 5.00 & & $7.5 \quad$ FLORA L, TREASLRE-(M) Clear pink .... \\
\hline \multirow[t]{3}{*}{1.00} & 7.50 & & *.9 FLoW'RET OF EIDEN-A beautiful rink. \\
\hline & 2.00 & $\$ 15.00$ & 8.6 FRANCES SIIAYLOR - Creamy white .......... \\
\hline & 2.00 & 15.00 & 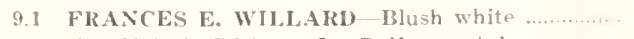 \\
\hline 10.00 & 100.00 & & FRANK E. GOOD -(L) Delicate pink …........... \\
\hline \multirow[t]{3}{*}{1.00} & 7.50 & & 8.2 FRANCOIS Rot'ssEAU-(E) Velvety red. \\
\hline & 2.00 & 15.00 & Q.2 GALATIIEE-Flesh white \\
\hline & 2.00 & 15.00 & 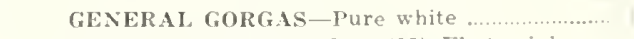 \\
\hline \multirow[t]{3}{*}{1.00} & 7.50 & & 8.9 GEORGIANA SIIAYLOR-(M) Flesh pink ........ \\
\hline & 2.35 & 17.50 & 8.5 GERMA1NE BIGOT-(M) Lilac-rose .......... \\
\hline & 2.00 & 15.00 & 8.2 GIGANTEA-(M) Bright pink , r..... \\
\hline \multirow[t]{4}{*}{1.00} & 7.50 & 50.00 & 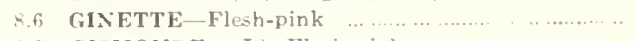 \\
\hline & 2.75 & 20.00 & 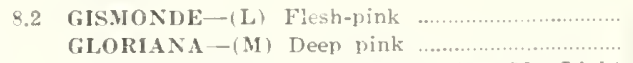 \\
\hline & $\begin{array}{l}2.75 \\
2.00\end{array}$ & $\begin{array}{l}20.00 \\
1.5 .00\end{array}$ & 7.9 GLORE DE CIIS. GOMBAULT (M) Light \\
\hline & $\begin{array}{l}2.00 \\
3.25\end{array}$ & $\begin{array}{l}15.00 \\
25.00\end{array}$ & $\begin{array}{c}\text { rose } \\
7.3 \text { GOLDEN HARVEST-(M) Blush pink }\end{array}$ \\
\hline \multirow[t]{4}{*}{1.00} & 7.50 & & 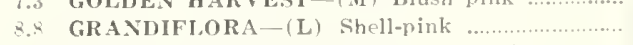 \\
\hline & & & 4.2 (iROVER ('IEVEIAXI) (L) Dark crimise... \\
\hline & 2.75 & 20.00 & 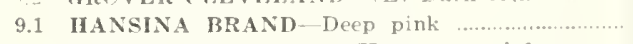 \\
\hline & 2.00 & 15.00 & 9.0 II AZEL liNNEY--(M) Hydrangea-pink \\
\hline \multirow[t]{2}{*}{6.00} & 60.00 & & 7.9 HELEN WOLAVER (L) Shell pink ........ \\
\hline & 2.00 & 15.00 & IIENRI CORE (L) Flesh pink .......... \\
\hline \multirow[t]{2}{*}{1.00} & 10.00 & & S,8 IIENRY IVERY-(I, Light pink \\
\hline & 2.00 & 15.00 & 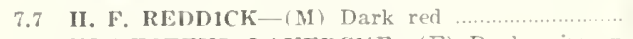 \\
\hline \multirow[t]{2}{*}{1.00} & 7.50 & & Q.5 INSPECTEUR LAVERGNE--(E) Dark crimson \\
\hline & 2.00 & 15.00 & 8.7 JAMES KELWAY-(M) Pure white...... \\
\hline \multirow[t]{2}{*}{2.00} & 20.00 & & $\times .3$ JEANNE GAUDICHAI - White ............ \\
\hline & 3.25 & 25.00 & 9.3 JOIIN M. GOOD-( 1 ) pure white... \\
\hline \multirow[t]{2}{*}{1.00} & 7.50 & & 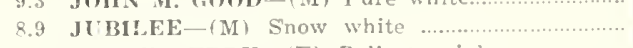 \\
\hline & 3.25 & 25.00 & Q.6 JUD(iE BERRI-(E) Delicate pink ...... \\
\hline 1.00 & 7.50 & & 8.8 KARL ROSEFIELD (M) Velvety crimson.... \\
\hline \multirow[t]{4}{*}{2.00} & 20.00 & & 9.8 KELWAI'S GLORIOI'S--Pure white ......... \\
\hline & 2.00 & 15.00 & 8.8 KELWAY'S QIEEN (L) Flesh pink ....... \\
\hline & 2.00 & 15.00 & 9.1 LADY ALEXANIDRA DIFF Blush pink \\
\hline & 2.00 & 15.00 & 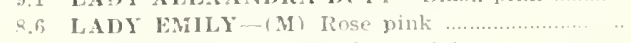 \\
\hline \multirow[t]{8}{*}{1.00} & 7.50 & & 7.8 LA FAYETTE-(M) Salmon-pink ............ \\
\hline & 2.00 & 15.00 & 9.2 LA FEE - (E) Guards-Mauve rose.. \\
\hline & 2.00 & 1.3 .00 & 9.0 LA FRANCE--(M) I La France pink ..... \\
\hline & 2.00 & 15.00 & 9.6 LA IORIAINCE (MI) Creamy-white \\
\hline & 2.01 & 15.00 & 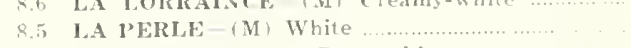 \\
\hline & 2.00 & 15.00 & 8.3 LA ROSIERE - (M) Pure white .............. \\
\hline & 2.90 & $1^{-}-00$ & S.1 1.A TFNDRESSE-(E) C'reniny-white ... \\
\hline & 2.75 & 20.00 & 7.5 LA TU1MPE-(M) Delicate blush-white \\
\hline 3.00 & 30.00 & & \& LAIRA DESGERT-(F) Canary-yellow \\
\hline $1.2 . j$ & 12.30 & & 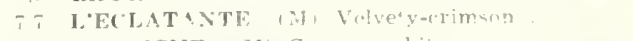 \\
\hline \multirow[t]{2}{*}{1.00} & 3.50 & & 9.9 LE CYCNE (M) Creany white ............... \\
\hline & 2.75 & 20.00 & Q LILLIAN G('MII Apple blossom nink... \\
\hline 1.75 & $17 . .50$ & & 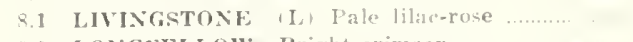 \\
\hline 1.00 & 7.50 & & a) LONGFELLOW-Bright crimson \\
\hline \multirow[t]{2}{*}{6.00} & 60.00 & & R.4 IOORA DEXIIEIMEI - F) Intense erimson \\
\hline & 2.75 & 20.00 & - LORO KITCHENER |E) (herry-ret ... \\
\hline 1.00 & 7.50 & & in IOIISA BRANI IL, Blush-whito ...... \\
\hline \multirow[t]{3}{*}{1.00} & $7 . .0$ & & X.T LIETTA PFEIFER - (E) Glistening white \\
\hline & 2.75 & 20.00 & \&.1 MADAMF (AIOT, H, IIvelrameril-pink ... \\
\hline & 2.00 & 15.00 & 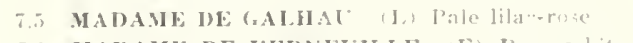 \\
\hline \multirow[t]{2}{*}{2.00} & 20.00 & & T.9 MADAME DE VERNEVILLE (E) I'ure whit \\
\hline & 2.00 & 15.00 & 7.9 MADAUE DUCEI- M) Silvery lilac-pink \\
\hline
\end{tabular}

$\begin{gathered}\text { Per } \\ \text { Each }\end{gathered}$

$\$ 5.00$
1.00
1.00
17.50
1.00
1.00
2.00
1.00

\begin{tabular}{c|c} 
Per & Per \\
I2 & I
\end{tabular}

$\$ 2.35 \$ 17.50$

$2.00 \quad \mathbf{I 5 . 0 0}$

50.011

7.50

7.50

7.00

7.50

7.50

20.00

7.50

2.00

3.2.5

1.00

8.00

2.75

2.35

2.00

2.75

.7 .3

$6.00 \quad 60.00$

$6.00 \quad 60.00$

$1.00 \quad 7.50$

$2.00 \quad 20.00$

$\begin{array}{ll}1.25 & 12.50\end{array}$

3.25

40.00

5.00

$1.00 \quad 7.50$
7.507

$7.50 \quad 75.00$

$1.00 \quad 7.50$

$1.00 \quad 7.50$

3.25

$1.50 \quad 15.00$

$2.50 \quad 25.00$

I.00 7.50

$1.00 \quad 7.50$

3.25

$1.50 \quad 15.00$

1.0010 .00

$2.00 \quad 20.00$

2.75

\subsection{0}

2.75

2.00

$1.25 \quad 12.50$

2.01

I.50, 15.00

$1.25 \quad 12.50$

2.00

$1.00 \quad 7.50$

$1.00 \quad 7.50$

$3.25 \quad 2.5 .00$

$3.25 \quad 3.50$

1.00

7.50

$2.00 \quad 15.00$

$2.00 \quad 1.5 .00$

$2.00 \quad 13.00$

$2.75 \quad 20.00$ 
General List of Double Peonies

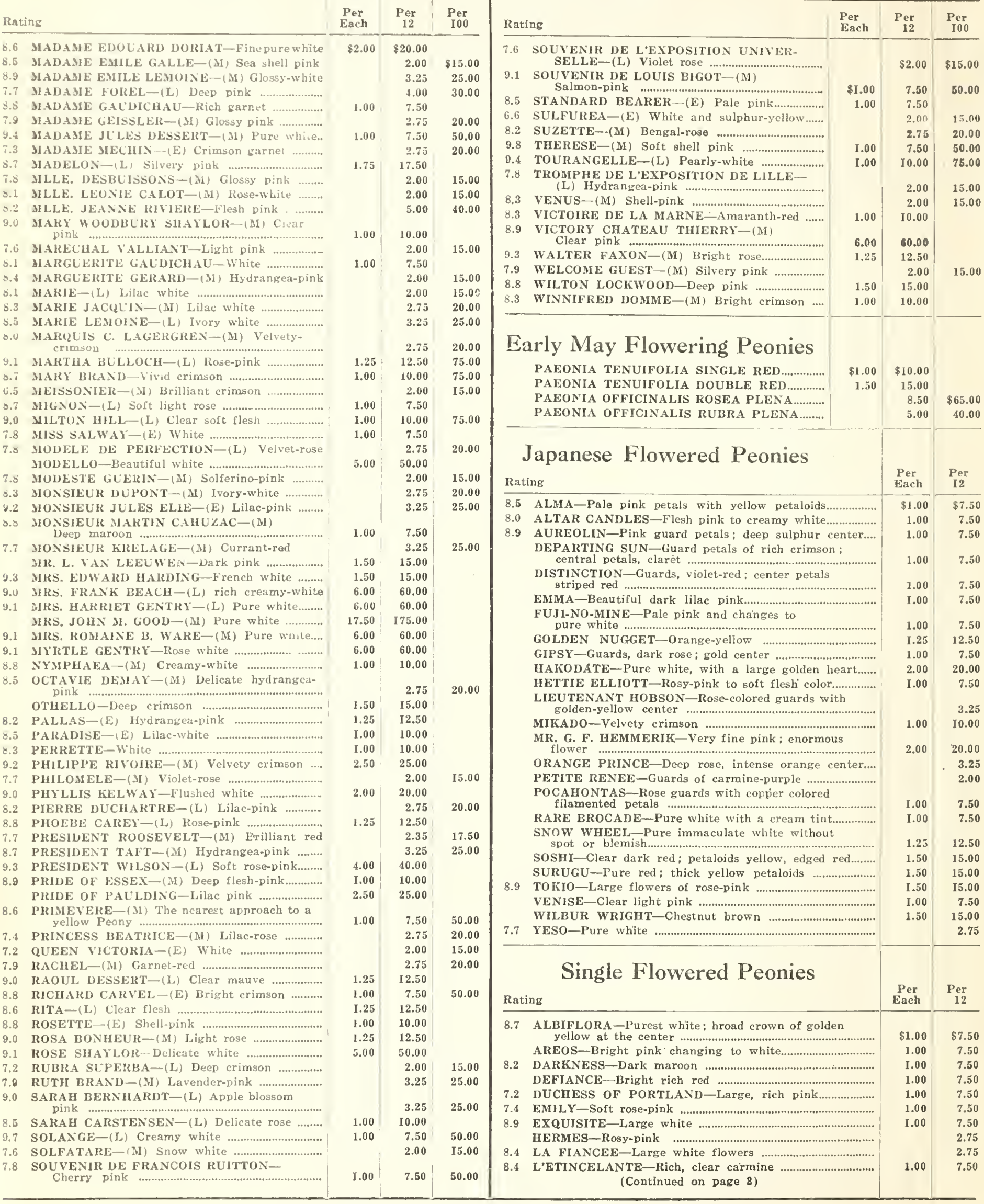


Single Flowered Peonies

(Concluded from page 2)

Rating

7.7 LUCIENNE-White, flushed purplish-carmine

MISTRAL-Very brilliant carmine

8.j ROSY DAWN-Day-break pink ....

8.0 THE MOOR-Rich, glowing crimson

VESUVE-Brilliant dark red

WAR-Large carmine red

\section{Peonies in Mixture}

\section{White in Mixture}

Red in Mixture.

Pink in Mixture

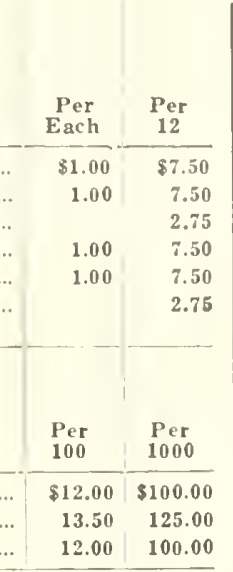

German Iris

$\$ 3.00$ per $100, \$ 20.00$ per 1000
Ime. Chereau

IIme. Pacquitte

Monsignor

Nicbelungen

Nokomis

Pallida Dalmatica

Perfection

Purple Prince

Quaker Lad.

Rhein Nixe

Shekinah

Shrewsbury

\section{German Iris}

$\$ 4.00$ per $100, \$ 30.00$ per 1000

Agnes

Annie Leslie

Archeveque

Armenian

Aurea

Australis

Azure

Belcolor
Blue Jay

Camelot.

Carthusian

Cecile Minturn

Chasseur

Cherubin

Clematis

Clio

Crusader
Dahlia

Dalmarius

Delicatissima

Demure

Dora Longdon

Dorthea

Grapta

Grevin

IIuber

Hugo

Jeanne d'Arc

Juniata

Kathryn Fryer

Inysna

La Neige

Lewis Trowbridge

Loreley
Major

Mandralisca

Margaret Moor

Marsh Marigold

Mary Garden

Mme. Cheri

Monsignor Boyer

Mrs. Cowley

Mrs. H. Darwin

Neptune

Nuee d'Orage

Pocahontas

Princess Victoria Louise

Queen Flavia

Queen of May

Rodney

Iromany

Romeo

Seminole

Sherwin Wright

Simonne Waissiere

Sunset

Swatara

Sweet Lavender

Syphax

Tom Tit

Trianon

Tristram

Trojan:

Turco

Violacea Grandiflora

Walhalla

White knight

Wyomissing

Yvonne Pelletier

Zwaventurt

\section{Fall Bulbs for Florists}

\begin{tabular}{|c|c|c|}
\hline $\begin{array}{l}\text { Breeder Tulips } \\
\text { Top Quality. }\end{array}$ & $\begin{array}{l}\text { Per } \\
100\end{array}$ & $\begin{array}{r}\text { Per } \\
1000\end{array}$ \\
\hline ALCIDA-Yellow and brown & $\$ 3.75$ & $\$ 35.00$ \\
\hline APRICOT-Bronzy huff ............. & 3.25 & 30.00 \\
\hline ASPASIA--Red shaded salmon & 3.75 & 35.00 \\
\hline 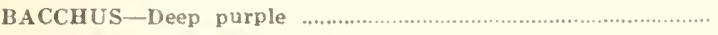 & 3.25 & 30.00 \\
\hline CARDINAL MANNING-Rose violet & 3.00 & 27.50 \\
\hline 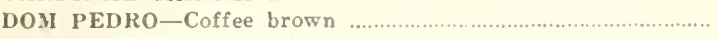 & 3.75 & 35.00 \\
\hline GRAND IAITRE-Purplish violet ... & 3.75 & 35.00 \\
\hline HAMLET-Yellowish bronze .............. & 3.25 & 30.00 \\
\hline JAUNE D'OEUF-Ruddy apricot & 3.25 & 30.00 \\
\hline 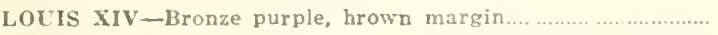 & 2.75 & 25.00 \\
\hline MARIA LOTISE-Salmon and orange............... & 3.2 .3 & 30.00 \\
\hline 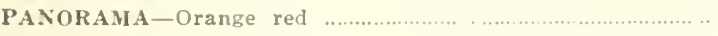 & 2.75 & 25.00 \\
\hline PRINCE OF ORANGE-Rich red .... & 3.25 & 30.00 \\
\hline PROF. SCFOTEL-Very good blue & 3.25 & 30.00 \\
\hline 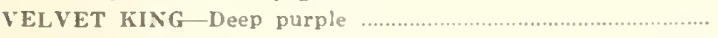 & 3.25 & 30.00 \\
\hline YELLOW PERFECTIONS-Bronzy yellow ............. & 3.25 & 30.00 \\
\hline 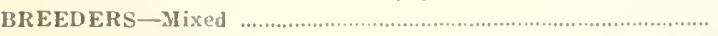 & 2.75 & 25.00 \\
\hline
\end{tabular}

\section{Single Early Tulips}

Top Quality.

CHRYSOLORA-Yellow

COTTAGE MAID-White

COULEUR CARDINAL-Scarlet

DUCHESS OF PARMA-Orange red

FLAMINGO-Dark pink

FRED MOORE-Bright orange

GEN. de WET-Orange yellow

IBIS-Carmine pink

KEIZERSKROON-Red and yellow

PRINCE OF AUSTRIA-Orange and scarlet

ROSE LUISANTE-Deep pink

WHITE SWAN-Pure white

WOLWERIAN-Rich purple

SINGLE EARLY UIXED

DarWin Tulips
Top Quality.

\section{Parrot Tulips}

Top Quality.

CHOICE HXED 
Fall Bulbs for Florists-(Concluded)

\begin{tabular}{|c|c|c|}
\hline Top Quality. & $\begin{array}{l}\text { Per } \\
100\end{array}$ & $\begin{array}{c}\text { Per } \\
1000\end{array}$ \\
\hline COURONXE D'OR-Orange yellow & $\$ 3.75$ & $\$ 35.00$ \\
\hline EL TOREADOR-Reddish orange & 3.75 & 35.00 \\
\hline 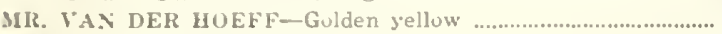 & 3.25 & 30.00 \\
\hline MURILLO-Light pink & 2.75 & 25.00 \\
\hline PEACH BLOSSOM-Dark pink & 3.25 & 30.00 \\
\hline 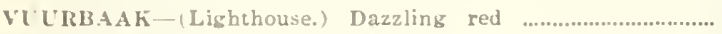 & 3.25 & 30.00 \\
\hline CHOICE MIXED-All colors & 2.75 & 25.00 \\
\hline
\end{tabular}

\section{Cottage Tulips (May flowering)}

Top Quality.

\section{ARGo-Golden yellow:}

ATIS KENNICOTT-Deep sellow

BOUTON D'OR-Pure yellow

DIDO-Orange pink

GESNERIANA SPATHULATA-Scarlct

GRENADIER-Orange scarlet

JOHN RCSkIN-Yellow and pink

MISS WILMOTT-Light yellow

PICOTEE-Blush pink

ROSAIBELLA-Bright pink

SIIXED -All colors

\section{Hyacinths}

First size $(18-19 \mathrm{~cm}$.)

\section{GERTRLUE-Deep rose}

GRAND MAITRE-Bright blue

LA VICTOIRE-Bright scarlet

L'INNOCENCE-Pure white

MARCONI-Red

\section{Hyacinths}

Single Hyacinth -2 nd size $(17-18 \mathrm{~cm}$.)

DR. LIEBER-Early; lavender violet

LA VICTOIRE-Bright scarlet

L'INNOCENCE-Pure white

MARCONI-Red with white shades

SCHOTEL-Forget-me-not hlue

\section{Single IHyacinths}

Third Size (I6-17 cm.)

GERTRLDE-Deep rase

GRAXI MAITRE-Bright porcelain hlue

KING OF THE BLUES-Deep blue

LADY DERBY-Delicate pink

L'INNOCENCE-Pure white

LA VICTOIRE-Bcautiful hright scarlet

IARCONI-Red with white shades..

QUEEN OF THE BLUES-Silvery blue

QUEEN OF TIIE PINKS-Brilliant pink

YELLOW IIAMMER-Pure yellow

MIXED -All colors

\section{Miniature Hyacinths}

\section{$(13-15 \mathrm{~cm}$.}

\section{GERTRUDE}

GRAND MAITRE

L'INNOCENCE

MARCONI

LA GRANDESSE

QUEEN OF THE BLUES

\section{Narcissus}

Washington Grown Bulbs

BARRII CONSPICUOUS-Yellow perianth, cup edged orange-scarlet; free flowing...

EMPEROR-Primrose perianth, rich yellow trumpet... EMPRESS-(Giant Trumpet)-White perianth, rich yellow trumpet ......................................................................

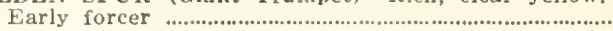

KING ALFRED (Giant Trumpet)-Intense golden yellow. ETICUS RECURVUS (Pheasant's Eye) - White, perianth with red eye....

SIR WATKINS (Medium Trumpet)-Sulphir-yellow, cup tinged with orange

SULPHUR PHOENIX (Double)—Creamy white, sulphuryellow center

VON SION (Douhle)-Deep golden yellow; good forcer...
$\$ 3.75 \$ 35.00$

$3.75 \quad 35.00$

$2.75 \quad 25.00$

$4.25 \quad 40.00$

$3.25 \quad 30.00$

$3.25 \quad 30.00$

$3.00 \quad 27.50$

$\mathbf{3 . 7 5} \quad \mathbf{3 5 . 0 0}$

$2.75 \quad 25.00$

$3.75 \quad 35.00$

$2.75 \quad 25.00$

25.00

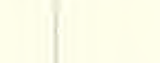

\begin{tabular}{l|l} 
Per & Per \\
12 & 100
\end{tabular}

\begin{tabular}{r|r}
$\$ 1.00$ & $\$ 6.50$ \\
1.00 & 6.50 \\
1.00 & 6.50 \\
1.00 & 6.50 \\
1.00 & 6.50 \\
\hline
\end{tabular}

\begin{tabular}{l|r}
\hline Per & Per \\
12 & 100 \\
\hline$\$ 0.85$ & $\$ 5.50$ \\
\hline 85 & 5.50 \\
.85 & 5.50 \\
.85 & 5.50 \\
.85 & 5.50 \\
\hline
\end{tabular}

$+5.50$

\begin{tabular}{|c|c|}
\hline $\begin{array}{l}\text { Per } \\
\text { Doz. }\end{array}$ & $\begin{array}{l}\text { Per } \\
100\end{array}$ \\
\hline$\$ 0.75$ & $\$ 4.50$ \\
\hline .75 & 4.50 \\
\hline .75 & 4.50 \\
\hline .75 & 4.50 \\
\hline .75 & 4.50 \\
\hline .75 & 4.50 \\
\hline .75 & 4.50 \\
\hline .75 & 4.50 \\
\hline .75 & 4.50 \\
\hline .75 & 4.50 \\
\hline .75 & 4.50 \\
\hline
\end{tabular}

.50

(n)

\section{Paper White Narcissus}

Well ripened, selected hulhs that may be expected to give the

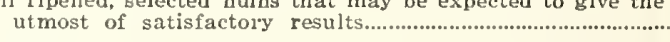

\begin{tabular}{|c|c|}
\hline $\begin{array}{l}\text { Per } \\
100\end{array}$ & $\begin{array}{c}\text { Per } \\
1000\end{array}$ \\
\hline$\$ 2.50$ & $\$ 20.00$ \\
\hline 5.50 & 50.00 \\
\hline 5.50 & 50.00 \\
\hline 5.00 & 45.00 \\
\hline 8.00 & 75.00 \\
\hline 2.50 & 20.00 \\
\hline 5.50 & 50.00 \\
\hline 4.00 & 35.00 \\
\hline 4.50 & 40.00 \\
\hline
\end{tabular}

\section{Freesias}

(August and Later Delivery)

Select Bulbs for Forcing

IMPROVED PURITY- $1 / 2$ to $5 / 8$ inch...

RAINBOW MIXTURE-All colors, 1/2 inch up.

\section{Crocus}

Choice Bulbs

NAMED VARIETIES-Separate colors

MIXED-All colors

\section{Oxalis}

Well-grown Bulbs

BERMUDA-Buttercup yellow

GRAND DUCHESS-Pink .

GRAND DUCHESS-Lavender

GRAND DUCHESS-White

\section{Hardy Lillies}

The Leading and Best Varieties for Forcing and Garden Planting

LILIUM AURATUM (The Gold Banded Lily of Japan)-

First size bulhs

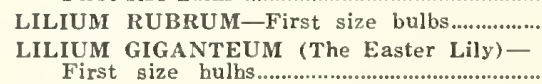

LILIUM CANDIDUM (The Madonna Lily)-

$20-22$ c. $\mathrm{m}$.

LILIUM REGALE (The Royal LiIy)-

First size bulbs.

\section{Good \& Reese Giant Hybrid Amaryllis}

These reselected hybrids are gigantic in size and exquisite in colorings. The colors range from white with thin markings of carmine, various shades of pink, to deep scarlet. Some are pure in color, others have heautiful markings and mottled throats. $\$ 4.00$ per $12, \$ 30.00$ per 100 .

\section{Good's White Giant Amaryllis}

Only once in a decade is a really worthwhile new plant produced. In Good's White Giant, we helieve we have a sensational new introduction that will hecomc as popular as Lilium Giganteum when its merits hecome known. The flowers are enormous in size, of ten ten inches in diameter. In color, pure white without markings of any kind on the flowers. Frequently as many as six flowers are carried on a single stem. It is the most striking flower that can he grown. The stock is limited this year. $\$ 1.00$ each, $\$ 7.50$ per $12, \$ 50.00$ per 100 . 


\section{Plants for Miniature Gardens!}

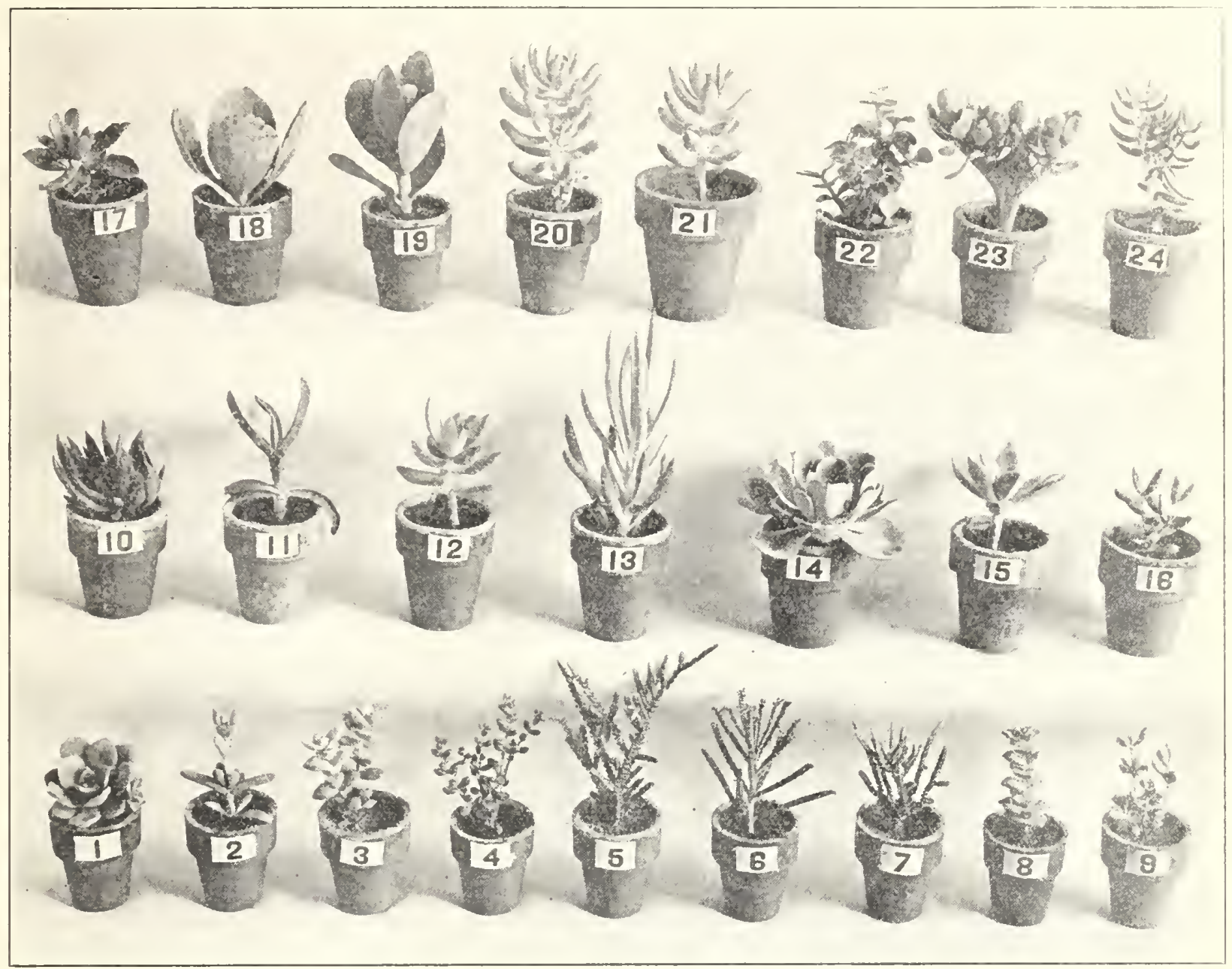

ANACAMPSEROS LANCELATA (No. 10)

ANTHERICUM VARIEGATUM ...................

ASPEDIUM FERN

BUXUS SEMPERVIRENS

CHINESE EVERGREEN

CHINESE EVERGREEN (Large)

CRASSULA ARBORESCENS, 3"

CRASSULA ARBORESCENS, 4

CRASSULA LYCOPODIODES ( $\mathrm{No}, 5)$

CRASSULA MONTICOLA (No. 3)

CRASSULA PSEUDOLYCOPODIODES (No,

CRASSULA TETRAGONA (No. 24)

CRYTOMIUM (Holly Fern)

ECHEVERIA SECUNDA GLAICA (No 14 )

ECHEVERIA WEINBERGII (No. 15) .....................

EPIPHYLLUM (Christmas Cactug)

EUONYMUS JAPONICA W....................

EUPHORBIA SPLENDE

FERNS-Dish Varieties

MOSS FERN-SELACINELLA

GREVILIEA ROBUST

HEXIA-IRISH MOSS or BA BY TE TEARS

ENGLISH IVY E....... ISH IVY

KALANCHOE FLAMMEA (No, 19)

KALANCHOE MARMORATA (No, 18)

KALANCHOE TUBIFOLIA (No.

KLEINIA FICOIDES (No 13)

MESEMBRYANTHEMUM DELTOIDES (No. 9)

MESEMBRYANTHEMUM AEQUILATERALA (No, 11)

MESEMBRYANTHEMUM ECHINATUM (No, 16)

MESEMBRYANTHEMUM

\begin{tabular}{r|r}
$\begin{array}{r}\text { Per } \\
12\end{array}$ & $\begin{array}{r}\text { Per } \\
100\end{array}$ \\
\hline$\$ 1.50$ & \multicolumn{1}{|c}{$\$ 10.00$} \\
.85 & 6.00 \\
.85 & 6.00 \\
.85 & 6.00 \\
1.50 & 10.00 \\
2.00 & 15.00 \\
.85 & 6.00 \\
1.75 & 12.00 \\
4.00 & 30.00 \\
.85 & 6.00 \\
1.50 & 10.00 \\
1.50 & 10.00 \\
.85 & 6.00 \\
.85 & 6.00 \\
.85 & 6.00 \\
1.20 & 8.00 \\
.85 & 6.00 \\
1.20 & 8.00 \\
.85 & 6.00 \\
1.20 & 8.00 \\
1.50 & 10.00 \\
.85 & 6.00 \\
.85 & 6.00 \\
.85 & 6.00 \\
.85 & 6.00 \\
.85 & 6.00 \\
1.50 & 10.00 \\
1.50 & 10.00 \\
1.50 & 10.00 \\
.85 & 6.00 \\
1.50 & 10.00 \\
.85 & 6.00 \\
1.50 & 10.00 \\
1.50 & 10.00 \\
1.50 & 10.00 \\
1.20 & 8.00 \\
& \\
\hline
\end{tabular}

OPHIOPOGON JAPONICA

OLEANDER

PACHYSANDRA TERMINALIS

PEPEROMIA OBTUSIFOLIA

SAXIFRAGA SARMENTOSA

SANSEIIEKIA ZEYLANICA

SANSEVIERIA ZEYLANICA

SANSEVIERIA LAURENTI

SANSEVIEIRA LAURENTI

SEDUM DENDROIDEUM CR

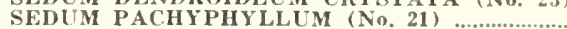

SEDUMI STAILII (No. 4)

SEDLM TRELEASEI (No. 20)

SEMPERVIVLM COOPERII (No. 17)

SEMPERYIVUM GLOBIFERA

SEMPERVIVUM HAWORTIII (No.............

SEIPERVIVIM TECTORLM

$\$ 0.85 \$ \$ 6.00$

$.85 \quad 6.00$

.856 .00

$.85 \quad 6.00$

$1.20 \quad 6.00$

$1.75 \quad 12.00$

1.00
1.50

$6.50 \quad 50.00$

$1.50 \quad 10.0$

$1.50 \quad 10.00$

$.85-6.100$

$\begin{array}{ll}.85 & 6.00 \\ .85 & 6.010\end{array}$

$1.20 \quad 8.01$

1.20

Realizing that many florists are unacquainted with most of these plants, we are offering three special collections of Miniature Garden plants. These collections include the best selling plants and are exceptional values in every particular.

$\$ 6.00$

$\$ 8.00$

$\$ 12.00$

In ordering, state Miniature Garden Collection. 


\section{Hardy Perennial Plants}

We are giving special attention to our Hardy Plant Department. Our stock is exceptionally large this year, one million plants being grown especially for the shipping trade. The majority of the perennials here offered are field-grown clumps.

ICIILI.F.A. The P'earl

AETIIONEMA I'ERSICLM

AGIROSTEMAIA CORUNAIIA

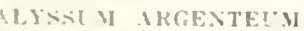

ALYSTLM IROATIR.ATL M

ALYSSIM SAXATILE COMIPCTIM

A.NCIITSA ITALICA, Dropmore

AQCILECIA I.Mrs. Scote Elliott IIybrids

AQL1LEGIA-Waysirle Pink

ARABIS ALPISA

ARENAIRIA MONTANA

AIRERIA MAITTIMA

ARMEIRIA HYIBIDS

AIRTFMISI.A SILVER KING

ASTEIR AFENIDROTIIE-Rosy, rer

A.TEIR CLIMAX IBlue

ASTEIR DELICATE-Lilac rose

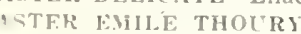

ASTER IIIGIILAND MOIRN-Blue

ISTEIR MAGr'E PERIRY-Mave

ASTER MONS Recklish violet

ISTEIR PERIRY'S BLUE-Blue

A.TER IROYAL BLCE-Rich blue

ASTER SAM BANHAM-White

ASTER ST. EGWIN-Light pink

ASTERS HARDY MIXD

ISTER NEW BABY'S BREATH

ASTILBE JAPONICA AMERICA

ASTILBE JAPONICA Gloria Superba

ASTILBE JAPONICA-Peachhlossom

IIBRETIA (Wall Cress)

I3.APTISIA ALSTIRALIS

IOLTONIA ASTEROIDES

BOLTONIA LATISQLAMA

( AMPANILA-Carpatica Blue

("AMPANI'LA-Carpatica Alha

('AMPACILA-Persicifolia Blue

('AMI'ATLA-Pyramidalis

("AMPANILA-Medium white

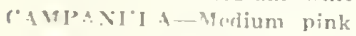

('APANLLA-Medium blue

('ENTAIREA MACROCEPHALA

('EVTAZIREA MONTANA-Blue

('ENTAYREA MONTANA-Aiba

('ERASTII'M TOYENTOSEM

CIIEIRANTHLS ALLIONI

CIRVSANTHEMTM COREANUM

COIROPSIS I.ANCEOLATA GRANDIFLORA

CIRTCIANELLA STVIOSA

DAISY IIART.IE \& ELDER

D.IISY AI.ASKA

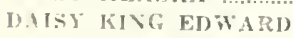

DELIIINILM BELI,ADONAA

DELPIIIITM BELLAMOSA

5) FIPIJIIT:I RT ACKMOIEF \& LANGDON (Hybrids)

I)IANTHI'S ('AESILS (Cheddar Pink)

DIANTHIS DELTOIDES

IIANTII'S IJ.IRBATI'S (Sweet William)-Salmon, white, rose, maronn

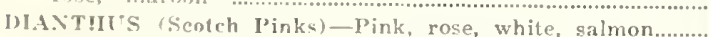

DICENTIRA SPECTAMILIS (Ilecding Heart)

I)IGITALIS (Foxglove)-White, purple, nink, yellow.

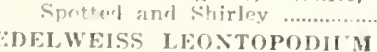

TIRIGERON SIDECIOSI'S IIVIRIIIS

FiRINIS ALISINA

IEI PATOLIIM COEIESTINIM (Ageratum)

FAILLARDIA GIRANIOIFLORA

CAILLAIRIA MAISY FEIRGISON

GET"M IADY STPATHEIEN

6.EIM MRS. BIRADSIIAW

(:TPCOPIILA IRISTOI, FAIRY

FYPSOPIILA PANICULATA

\begin{tabular}{|c|c|}
\hline $\begin{array}{l}\text { Per } \\
12\end{array}$ & $\begin{array}{c}\text { Per } \\
\text { I } 00\end{array}$ \\
\hline$\$ 1.00$ & $\$ 7.00$ \\
\hline 2.00 & 15.00 \\
\hline I. .00 & 7.00 \\
\hline 1.00 & 7.00 \\
\hline 1.00 & 7.00 \\
\hline 1.00 & 7.00 \\
\hline 1.00 & 7.00 \\
\hline I. .00 & 7.00 \\
\hline 1.00 & 7.00 \\
\hline 1.00 & 7.00 \\
\hline 1.00 & 7.00 \\
\hline 1.00 & 7.00 \\
\hline 1.00 & 7.00 \\
\hline 1.00 & 7.00 \\
\hline 1.00 & 7.00 \\
\hline $\mathbf{I . 0 0}$ & 7.00 \\
\hline I. .00 & 7.00 \\
\hline 1.00 & 7.00 \\
\hline I.00 & 7.00 \\
\hline 1.00 & 7.00 \\
\hline 1.00 & 7.00 \\
\hline 1.00 & 7.00 \\
\hline 1.00 & 7.00 \\
\hline 1.00 & 7.00 \\
\hline I.00 & 7.00 \\
\hline .85 & 6.00 \\
\hline I.50 & I 0.00 \\
\hline 3.25 & 25.00 \\
\hline 4.00 & 30.00 \\
\hline 3.25 & 25.00 \\
\hline 1.00 & 7.00 \\
\hline 1.20 & 8.00 \\
\hline 1.00 & 7.00 \\
\hline I.00 & 7.00 \\
\hline I.50 & 10.00 \\
\hline 1.50 & 10.00 \\
\hline I.50 & 10.00 \\
\hline 1.50 & 10.00 \\
\hline 1.00 & 7.00 \\
\hline 1.00 & 7.00 \\
\hline 1.00 & 7.00 \\
\hline 1.00 & 7.00 \\
\hline 1.00 & 7.00 \\
\hline 1.00 & 7.00 \\
\hline 1.00 & 7.00 \\
\hline $\mathrm{I} .00$ & 7.00 \\
\hline $\mathrm{I} .00$ & 7.00 \\
\hline 1.00 & 7.00 \\
\hline 1.00 & 7.00 \\
\hline .75 & 5.00 \\
\hline 1.00 & 7.00 \\
\hline 1.00 & 7.00 \\
\hline I.00 & 7.00 \\
\hline 1.00 & 7.00 \\
\hline 1.20 & 8.00 \\
\hline I.50 & 10.00 \\
\hline 1.00 & 7.00 \\
\hline 1.00 & 7.00 \\
\hline .85 & 6.00 \\
\hline 2.75 & 20.00 \\
\hline 1.00 & $7.0^{n}$ \\
\hline 2.00 & I 5.00 \\
\hline I. 50 & 10.00 \\
\hline 2.00 & 15.00 \\
\hline 1.00 & 7.00 \\
\hline I.00 & 7.00 \\
\hline 1.50 & I 0.00 \\
\hline 1.00 & 7.00 \\
\hline 100 & 7.00 \\
\hline 3.50 & 25.00 \\
\hline 1.00 & 7.00 \\
\hline
\end{tabular}

GYPSOPHILA REPENS

HELIANTIIEMUM IUTABILE

HELIANTHUS LARGE HYBRIDS

HELIOPSIS PITCHERIANA

HEMEROCALLIS FLAVA

IIERACLEUH VALLOSUM (Parsnip)

HEVCHERA SANGUINEA

HIBISCUS MALLOW MARVELS Pink, red, crimson-eye,

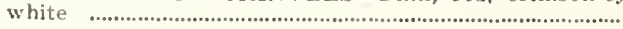

HOLLYHOCKS, Chater's Double Sorts-White, yellow,

crimson, salmon, rose

IBERIS GIBRALTARICA

IBEIRIS SEMIPERVIRENS
INCARVILLEA DELAVAYI

INCARVILLEA GRANDIFLORA BREVIPES

INULA ENSIFOLIA

LATHYRIS LATIFOLIA-(Red, pink, white)

LIATRIS PYCNOSTACIIY

LINUM PEIRENNE

LOBELIA C.ARDINALIS

LYCHNIS CHALCEDONICA

LICHNIS VISCARIA SPLENDENS

LYTHRUM ROSEUM SUPERBUM

MONAIZDA DIDYMA SCARLET

MYOSOTIS PALUSTRIS SEMPERFLORENS

OENOTHERA MISSOURIENSIS

NEPETA MUSSINI

NEPETA SOUV, de A. CHAUDRON

NEPETA ITRANA

\begin{tabular}{|c|c|}
\hline $\begin{array}{r}\text { Per } \\
12\end{array}$ & $\begin{array}{l}\text { Per } \\
100\end{array}$ \\
\hline$\$ 1.00$ & $\$ 7.00$ \\
\hline I .00 & 7.00 \\
\hline $\mathrm{I} .00$ & 7.00 \\
\hline 1.00 & 7.00 \\
\hline$I .00$ & 7.00 \\
\hline 1.50 & I 0.00 \\
\hline 2.00 & 15.00 \\
\hline I.00 & 7.00 \\
\hline 1.00 & 7.00 \\
\hline 1.00 & 7.00 \\
\hline 1.00 & 7.00 \\
\hline 2.00 & 15.00 \\
\hline 3.25 & 25.00 \\
\hline 2.00 & 15.00 \\
\hline 1.00 & 7.00 \\
\hline $\mathbf{I . 0 0}$ & 7.00 \\
\hline 1.00 & 7.00 \\
\hline 1.00 & 7.00 \\
\hline I.00 & 7.00 \\
\hline 1.00 & 7.00 \\
\hline I. 00 & 7.00 \\
\hline 1.00 & 7.00 \\
\hline 1.00 & 7.00 \\
\hline I.50 & 10.00 \\
\hline 1.00 & 7.00 \\
\hline 1.00 & 7.00 \\
\hline 1.00 & 7.00 \\
\hline
\end{tabular}

\section{Hardy Phlox}

$\$ 1.00$ per $12, \$ 6.50$ per $100, \$ 60.00$ per 1000 . Athis, Bridesmaid, Eclaireur, Isabey, La Vague, Mrs. Chas. Dorr, Mrs. Jenkins, Prime Minister, R. P. Struthers, Rheinlander, Riverton Jewel, Rosenberg, Rhynstrom, and Terra Neuva.

$\$ 1.10$ per $12, \$ 7.50$ per $100, \$ 70.00$ per 1000 . Beacon, B. Compte, Coquelicot, Enchantress, Europa, Gen. Von Heutz, Minnie West, Miss Lingard, Special French, and Thor.

$\$ 1.25$ per $12, \$ 8.50$ per $100, \$ 80.00$ per 1000 . Dr. Charcot, Firebrand, Gruppenkonigan, and Miss Ethel Pritchard.

PHYSALIS FRANCHETTI (Chinese Lantern Plant)

PHYSOTEGIA VIRGINICA

PIIYSOTEGIA VIRGINICA - Alba

PHYSOTEGIA VIRGINICA-Vivid

PLATYCODON MARIESII-Blue

PLATICODON MARIESII-Alba

PLATYCODON GRANDIFLORA-Blue

\begin{tabular}{|l|l|}
\hline 1.00 & 7.00 \\
\hline I.00 & 7.00 \\
\hline I.00 & 7.00 \\
1.20 & 8.00 \\
\hline I.00 & 7.00 \\
1.00 & 7.00 \\
\hline I.00 & 7.00 \\
\hline
\end{tabular}

\section{New Oriental Poppies}

FLANDERS-Oxblood red

LULA A. NEELEY-Oxblood red

MIRS. PERRY-Clear pink .

PROSERPINE-Brilliant scarlet

TRILBY-Cerise searlet

WURTEMBERGIA-Brilliant scarlet

GOOD \& REESE ORIENTAL HYBRIDS

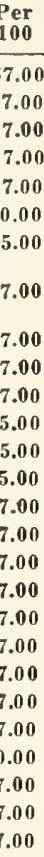



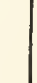




\section{Hardy Perennial Plants (Concluded)}

PLUMBAGO LARPENTAE POTENTILLA FRAGRIFORMIS POTENTILLA RECTA MACRANTHA PRIMLlA VERIS HYBRIDS

\section{New Double Pyrethrums \\ Wonderful for cut flowers}

MIAMI QTEEY-Large pink

BICKEYE-Large carmine

TROJAN-Dark pink

\section{$\$ 2.00 \$ \$ 15.00$ \\ $2.00 \quad 15.00$ \\ $2.00 \quad 15.00$}

PYRETHRTM GIANT HYBRIDS

RUDIBECKIA PURPLREA

SALVIA TIRKESTANICA

SCABIOSA IIOL'SE'S HYBRINS

SCABIOSA CALTCASICA

SCABIOSA CALCASIA Alba

SEDLM ACRE

SEDUM ALBLM

SEDLM ALTISSIMA HYBRIDT

SEDUM CORSICUM

SEDLM FOSTERIANA

SEDUM GLAICL'M

$\begin{array}{rr}\$ 1.00 & \$ 7.00 \\ 1.00 & 7.00 \\ 1.20 & 8.00 \\ 2.00 & 15.00 \\ 1.50 & 10.00 \\ 1.50 & 10.00 \\ .75 & 5.00 \\ .75 & 5.00 \\ 1.00 & 7.00 \\ 1.00 & 7.00 \\ .75 & 5.00 \\ .75 & 5.00\end{array}$

\section{Plants and Bulbs}

For Greenhouse and Nursery Planting

All plants from $2^{1}$, inch pots unless otherwise stated.

ACALYPHA SANDERII- (Chenille Plant)

AGERATUM FRASERI

A LOYSIA CITRIODORA- (Lemon Verbena)

ABELIA GRANDIFLORA

AHPELOPSIS VEITCIII-(Boston Iry)

ANTHERICEM VITATIM VARIEGATLM

ARTEMISIA SILVER KING

ASPARAGLS PLLMIOSI'S NANLS

ASPARAGUS SPRENGERII ...............

BOIGAINTILLEA SANDERIANA

BROWALLIA GIGANTEA Blue

BIDDLEIA ASIATICA

BLDDLEIA MAGNIFICA

CALI.A GODFREY

CLEMATIS PANICLLATA

CINERARIA GRANDIFIORA MAXIMA

COLEL'S CHRISTMAS GEMI-Rooted ruttings

COLEUS CIIRISTHAS GEM-2I inch pots

COLELS C. W. SCIIUIDT-(New) rooted cutting

COLEUS C. W. SCHMUT-21/4 inch pots

CYPERL's (I'mbrella Plant)

CLPHEA-(Cigar Plant)

IULSTY MILLER - Bush

DL'STY MILLER Trailing

DRACAENA INIDIVISA

ELONTMOL'S JAPONICA-IGreen)

EIONYMOL'S JAPONICA VARIEGATA

ETONYMOI'S RADICANS COIORATA

EPIPHYLLIN TRINCATIM-(Xmas Cacius)

ERLANGEA TOMENTOSA

FARFLGIUM GRANDE (Leopard PIant)-3" pots

GREVIJLEA ROILSTA

HELIOTROPE FLORENCE NIGHTINGAIA

IMPATIENS-Vermilion, salmon, red, white.

JAPANESE KUDZU VINE

NiEREMBERGIA AZIREA (IBue FIax)

NANUINA DOMESTICA

OLEANDER - Double red, vellow, pink, white

PACIISANDRA TERMINAIIS

PEPEROHIA (Watermelon Begonia)

PHILODENDRON CORIATIM

$\$ .00$

6.50

25.00

5.00

5.00

5.00

\begin{tabular}{|c|c|}
\hline $\begin{array}{c}\text { Per } \\
12\end{array}$ & $\begin{array}{c}\text { Per } \\
100\end{array}$ \\
\hline$\$ 1.00$ & $\$ 7.00$ \\
1.50 & 10.00 \\
1.50 & 10.00 \\
1.00 & 7.00 \\
\hline
\end{tabular}

SEDIM LINEAIR VARIGGATA

SEDL II LYCOPOJDES

SEDUM MIDDENDORFIANLM

SEDIM IREFLEI'M CIRISTATIM

SEDL I RUPESTRE

SEDLM SARMENTOSLN

SEDLM SIEBOLDI

SEDLM SPECTAIBILE

SEDLM SPECTAIBLE IBIIILLIANT

SEDLMI SPLRIUM

SEMPERTIVM GLOBIEERL M

SEMPERVIVIM TECTORIM

SIDALCEA SLSSEX BEACTY

SIIALCEA LOWFIELD--T'ink

STATICE LATIFOLIA

STOKESIA CYANEA-Blue

STOKESIA CYANEA-Alba

TIIALICTRI'M DIPTEROCARII II

TIRITOMA PFITZERI

TRITOMA GOOD \& REESE IIYBRIJS

TUNICA SAXIFRAGA

VALERIANA RLBRUM

VEIRONICA INCANA

VERONICA SPICATA

VERONICA LONGIFOLIA SL BSESAILIS

VIOLA SLTTON'S APRICOT

VIOLA JERSEY GEM

VIOLET PRINCESS OF WALES

IICCA FILAMENTOSA .............

\section{Plants and Bulbs--(Concluded)}

PILEA (Artillery I'Iant)

IRUELLIA MIOKOYANA

IRLSSELLIA ELEGANTISAIMA (Scarlet Fountain)

SAGE, IIOLT'S MAMMOITH

SAXIFRAGA (Strawberry Geranium)

SENECIO SCANDENS (Jarlor Ivy)

WANIDEING JEW-Bicolor and Tricolor

\section{Hardy Climbing Roses}

Strong 2-Year. Field-Grown Plants for Delivery

$$
\text { after November } 1 \text { st }
$$

IMERICAN PILLAR-Brilliant pink

CIIAPLIN'S PINK CLIMIBER (New)-Clenr pink

CLIMBING AMEIRICAN BEAUTY-Crimson

DOROTII PERKINS-Soft shell pink..

OR. W. VAN FIEET-Pale pink.

E.IILY GRAY-Bright yellow

EUGENE JACQUET-Bright cherry red.

EXCELSA Red Dorothy Perkins

GARDENIA-Creamy vellow

IIIA WTIIA-Dark rosy red

JACOTTE (New)-Orange yellow

MADAME GREGOIRE STAECIIELIN (New)-Pale pink

MAIR LOVETT-Pure snow white.

MARIE GOLCHALLT-Iight red

PAUL'S SCARLET CLIMIBER Vivid scarlet

ROSEIRIE T'rich't rose pink

SIIOWER OF GOLD - Bright yellow

ill Ter MOON-Snot: white

\begin{tabular}{c|c}
$\begin{array}{c}\text { Per } \\
12\end{array}$ & $\begin{array}{c}\text { Per } \\
100\end{array}$ \\
\hline$\$ 0.75$ & $\$ 5.00$ \\
.75 & 5.00 \\
.75 & 5.00 \\
.75 & 5.00 \\
.75 & 5.00 \\
.75 & 5.00 \\
.60 & 4.00
\end{tabular}

\section{Roses}

We will have our usual supply of Field Grown Budded lioses and Oun loot Pot Grown Plants for winter and spring delivery. Send your list of requirements for special quotations. 


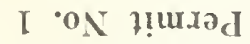

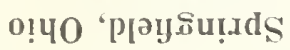

\section{CIVd}

ADYLSOd $\mathrm{S} \cdot \mathrm{C}$

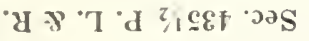

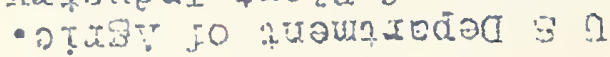

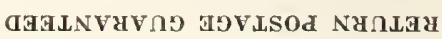

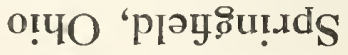

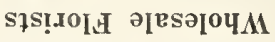 \\ .}

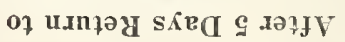

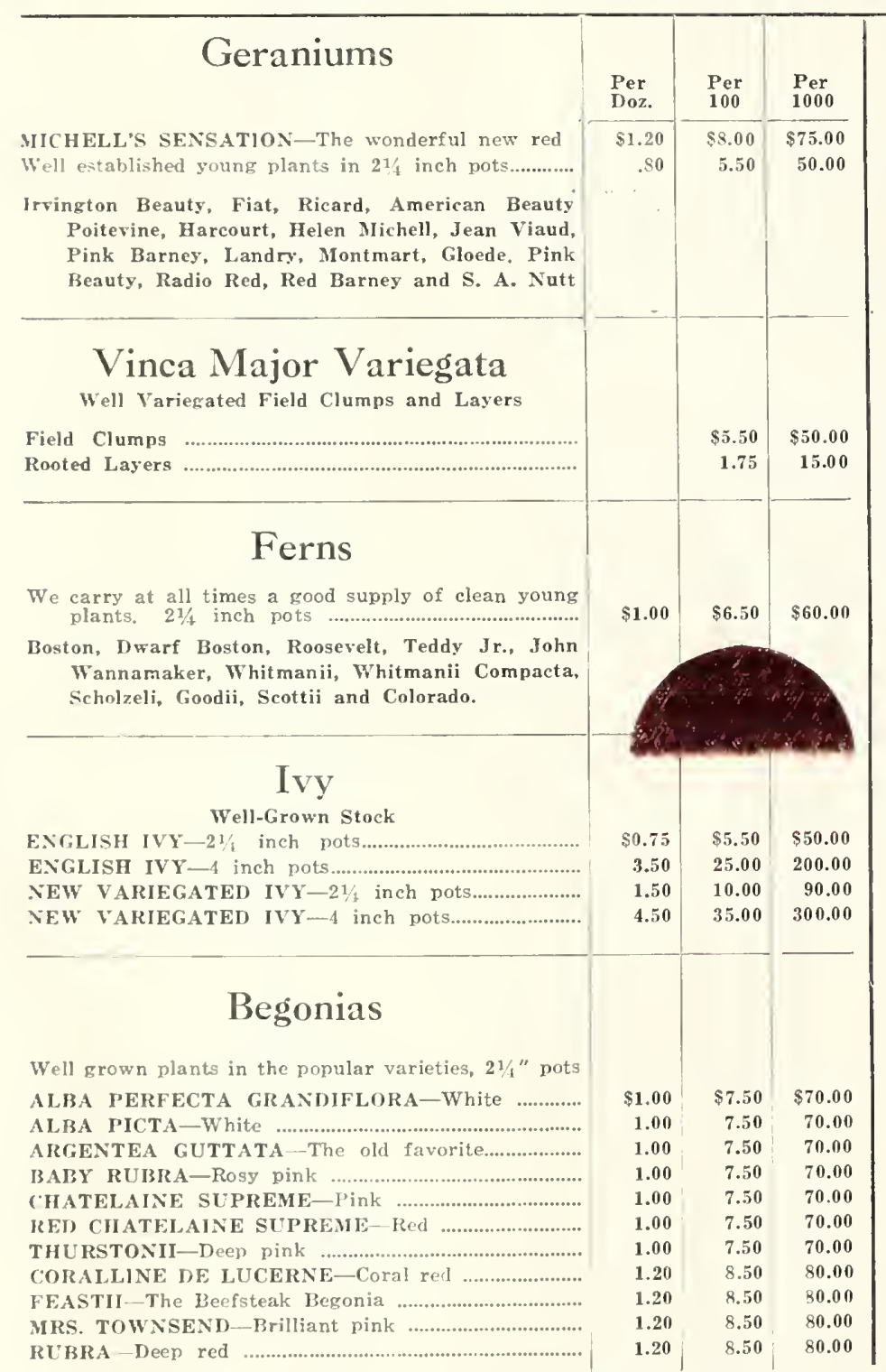

\section{Lantanas}

One hundred thousand choice plants, 21/4 inch pots...

Alba Perfecta, Craigi (new red), Biencourt, Golden Queen, Jacob Schulz, La Pleur d'Or, M. Schmidt, Radiation, Southern (new pink and yellow), Tethys, and Weeping.

\section{Sansevierias}

All pot grown greenhouse plants. Much superior to the bare rooted plants usually offered. SANSEVIERIA ZEYLANICA - 21/1/ inch pots SANSEVIERIA ZEYLANICA - 3 inch' pots.

SANSEVIERIA ZEYLAN1CA - 4 inch pots. SANSEVIERIA LAURENTI-3 inch pots..

SANSEVIERIA LAURENTI-4 inch pots.

SANSEVIERIA LAURENTI-5 inch pots.

\section{Hydrangeas}

Pot grown plants in the best forcing varieties

3 inch pots

4 inch pots

5 inch pots

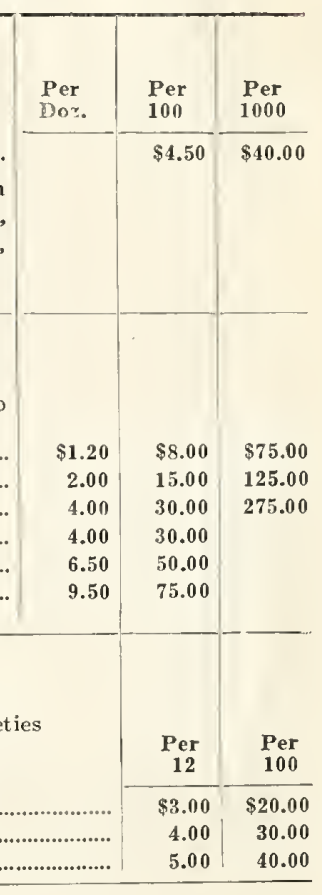

\section{Rex Begonias}

We offer the most highly colored varieties, $2 \frac{1}{2}$ inch pots, $\$ 1.50$ per $12 ; \$ 10.00$ per $100 ; \$ 90.00$ per 1000 .

\section{Primula Malacoides Erikssonii}

The best new pot plant novelty introduced in years. We offer three separate shades. $2 \frac{1}{4}$ inch pots, $\$ 1.10$ per $12 ; \$ 7.50$ per $100 ; \$ 70.00$ per 1000 .

\section{Saintpaulia-The African Violet}

We offer choice plants of this wonderful pot plant. 21/4 inch pots, $\$ 2.75$ per $12 ; \$ 20.00$ per 100 .

\section{Primula Obconica}

The famous $M$ and $M$ Strain. $2 \frac{1}{4}$ inch pots, $\$ 0.85$ per $12 ; \$ 6.00$ per 100 ; $\$ 50.00$ per 1000 . 